\title{
Nutritional supplements and herbal medicines for women with polycystic ovary syndrome; a systematic review and meta-analysis
}

\author{
Susan Arentz ${ }^{1 *}$, Caroline A. Smith ${ }^{1}$, Jason Abbott ${ }^{2}$ and Alan Bensoussan ${ }^{1}$
}

\begin{abstract}
Background: Polycystic ovary syndrome (PCOS) is a common, reproductive endocrinopathy associated with serious short and long term health risks. Many women with PCOS use ingestible complementary medicines. This systematic review examined the effect on menstrual regulation and adverse effects from randomised controlled trials.

Methods: Randomised controlled trials (RCTs) that compared herbal or nutritional supplements to placebo or active controls in women with PCOS were eligible for inclusion. Electronic databases were searched to July 2017. Study selection and assessment of quality were conducted independently by two review authors.

Results: Twenty four studies (1406 women) investigating seven nutritional supplements and four herbal medicines were included. No one study was assessed as having a low risk of bias. Four trials reported on the primary endpoint menstrual regulation. There was no evidence on improved menstrual regularity for calcium plus vitamin D compared to Metformin (RR: $0.66,95 \% \mathrm{Cl} 0.35$ to 1.23, $p=0.19$ ), reduced amenorrhoea for Camellia sinensis compared to placebo (RR: $0.17,95 \% \mathrm{Cl}$ 0.02 to $1.72, p=0.13$ ) and no difference in the number of menses per month for Cinnamomum sp. against placebo (MD $0.05,95 \% \mathrm{Cl}-0.36$ to $1.36, p=0.26)$.

Adverse effects were investigated in seven studies (164 women). Mild adverse effects were found for Cinnamomum sp. compared to placebo (17 women, RR: $0.36,95 \% \mathrm{Cl} 0.03$ to $0.70, p=0.03$ ). No difference was found for adverse effects between inositol, B complex vitamins, vitamin D, chromium and placebo. Improved reproduction, metabolic hormones and hyperandrogenism was found for inositol and improved cholesterol for omega three fish oils.
\end{abstract}

Conclusion: There is no high quality evidence to support the effectiveness of nutritional supplements and herbal medicine for women with PCOS and evidence of safety is lacking. High quality trials of nutritional supplements and herbal medicines examining menstrual regulation and adverse effects in women with PCOS are needed.

Keywords: Polycystic ovary syndrome, PCOS, Supplements, Vitamins, Herbal medicine, Complementary medicine

\section{Background}

Polycystic ovary syndrome (PCOS) is the most common female reproductive endocrine disorder [1] estimated to affect up to one in five women [2]. PCOS has broad health implications including adverse metabolic (obesity, type two diabetes, cardio vascular disease), reproductive (infertility, miscarriage, pregnancy and neonatal complications)

\footnotetext{
*Correspondence: s.arentz@westernsydney.edu.au

${ }^{1}$ National Institute of Complementary Medicine, Western Sydney University, Locked Bag 1797, Penrith, NSW 2751, Australia

Full list of author information is available at the end of the article
}

$[3,4]$, and psychological (anxiety, depression and stress) $[5,6]$ risks. The pathogenesis is underpinned by insulin resistance [7] which affects up to $75 \%$ of lean women and up to $95 \%$ of obese women (compared to $62 \%$ in BMI matched non-POCS controls) [8]. Prevention and reduction of being overweight with lifestyle interventions is first-line evidence-based treatment [9], however the strength of evidence for lifestyle is limited by high attrition in randomised controlled trials (RCTs) $[10,11]$ and, as in the general population, engagement and adherence to lifestyle intervention is impacted by psychosocial and physical 
barriers in women with established obesity [10]. Additional treatments for PCOS symptoms include pharmaceutical agents [9] (contraceptive pills (OCP) and hypoglycaemics), but contraindications for the OCP are common in overweight women [12] and hypoglycaemic agents are associated with significant rates of unpleasant side effects $[13,14]$, potentially worsening women's quality of life. Women with PCOS have been shown to seek out alternative [15] and adjunct treatments including complementary medicines $(\mathrm{CM})$ to improve their health, fertility and wellbeing [16].

There is some evidence of positive effects in women with PCOS for ingestible CMs including nutritional supplements $[17,18]$ and herbal medicines $[19,20]$. Women with PCOS have significantly higher levels of homocysteine and oxidative stress [21] and nutritional supplements have demonstrated efficacy in women with PCOS [18] and risk reduction for the same conditions in other populations [22]. Endocrine mechanisms of herbal medicine may improve hormone balance in PCOS and may positively effect menstural regularity [23, 24], a critial outcome of concern for women with PCOS [25]. This study aimed to synthesise the evidence of effectiveness and safety for herbal and nutritional supplements in women with PCOS.

\section{Methods}

RCTs that compared herbal medicines or nutritional supplements to placebo, active controls (nutritional supplements) or usual care (pharmaceutical treatment, lifestyle management) were sought. Cross-over trials were included for completeness but data from the first phase only were included in meta-analyses.

Participants were women aged between 18 and 44 with a diagnosis of PCOS according to the Rotterdam Criteria (2003), [26] or the National Institute for Health (NIH) [27].

Nutritional or herbal supplements were defined as herbal extracts in a single preparation and/or tablets containing vitamins, minerals or dietary derived molecules designed to improve symptoms associated with PCOS and not medically prescribed for conditions of deficiency [28].

The primary outcome measures were; 'menstrual regularity' defined as number of women with menstrual cycles of 22 to 35 days duration, number of women with amenorrhoea and number of menses per month, and 'adverse effects'. Secondary outcomes were; secondary menstrual characteristics (ovulation, presence of a dominant follicle, ratio of luteal weeks, gonadotropin hormones), polycystic ovaries, pregnancy rates and outcomes (live births and miscarriage), hyperandrogenism (clinical and biochemical), anthropometric factors, metabolic biochemistry, PCOS associated risk factors, quality of life and anxiety and depression.
All published and unpublished RCTs comparing herbal medicine or nutritional supplements with active controls, usual care or placebo, in English language, were searched. Electronic databases included Cochrane central register of controlled trials (CENTRAL), the Cochrane Library, MEDLINE ovidSP, CINAHL, SciVerse, EMBASE, PubMed were searched from the date of inception to July 2017. Reference lists of review articles and texts were searched by hand.

Keywords were 'polycystic ovary syndrome', 'PCOS', 'ovarian cysts,' 'hyperandrogenism,' 'hirsutism, 'acanthosis nigrans', 'acne,' 'oligomenorrhoea, 'amenorrhoea', 'oligoovulation,' 'anovulation', 'menstrual disorders', 'menstrual', 'ovulation,' 'fertility', AND 'alternative, 'integrative medicine', 'complementary medicine', 'naturopathy', 'natural', 'herbal medicine', 'botanical medicine' 'phytomedicine', 'phytotherapy, 'herbs,' 'diet', 'food', 'nutritional', 'nutrient', 'micro, 'dietary', 'vitamin', 'mineral', 'supplements', 'hypervitamin', AND 'randomis(z)ed. control trial', 'clinical trial' and 'RCT'.

Study characteristics, risk of bias and outcome data were manually extracted independently by two (of three) authors (CAS, JAA and SA) and entered onto data extraction forms adapted from the Cochrane Collaboration [29] for systematic critical appraisal. Differences of opinion were resolved by consensus. Risk of bias was assessed as high, low or unclear risk for randomization (generation of randomized schedule and allocation sequence), performance (blinding of participants), detection (blinding of outcome assessors and data analysts), attrition (reporting missing data), reporting (pre-specified and or relevant outcomes) and other areas of potential bias including factors unique to particular settings (such as study being stopped early, apparent fraudulent reporting (including the absence of declaration of funding and conflicts of interest in relation to authors affiliations) or extreme baseline imbalance) [29].

Analyses were conducted using Review Manager (RevMan) 5.3 [30]. Dichotomous data were expressed as relative risks (RR) and normally distributed continuous data as mean differences (MD). Corresponding 95\% confidence intervals (CIs) and $p$ values were calculated. Heterogeneity between studies was investigated by the $\mathrm{I}^{2}$ statistic $\left(\mathrm{I}^{2}\right.$ of more than $50 \%$ was considered indicative of heterogeneity), the $P$ value from the $\chi^{2}$ test and by visual inspection of the forest plots. For non-significant heterogeneity, results were pooled in a fixed effect model and the effects were adjusted using random effects where there was significant heterogeneity. If distributions were skewed and results reported as the median and range, with non-parametric tests of significance, the results were excluded from the metaanalysis. Androgens, cholesterol and triglycerides were converted to common measurements for comparison and the mean difference (MD) was calculated. 


\section{Results}

A total of 116 records were identified through the electronic and manual searches. Following initial screening, 63 articles were retrieved for detailed evaluation (Fig. 1). Thirty two full text articles were excluded as they did not meet the inclusion criteria (detailed in Additional file 1: Table S1). Following detailed evaluation of 31 full text studies, a further seven studies were excluded due to not investigating key signs and symptoms of PCOS, not being randomised or not including participants with a clinical diagnosis (detailed in Additional file 1: Table S1). Twenty four randomised controlled trials met the inclusion criteria [31-54] including 1406 women.

\section{Included studies}

\section{Design}

The sample sizes ranged from 10 [43] to 283 [37]. All studies were conducted at university hospitals or clinical research centres in America [42, 43, 46, 51, 52], Australia [35], Egypt [49], Hong Kong [33], Iran [32, 40, 44, 47, 48, 50, 54], Italy [31, 34, 36-38], Turkey [53], United Kingdom [39] and Venezuela [41, 45].

\section{Participants}

Five studies included overweight participants (BMI greater than 25) $[33,35,44,45,51]$, and two studies included women of normal bodyweight $[36,41]$. One study stratified randomization according to BMI (overweight or normal weight) [47]. Seventeen studies excluded participants with other endocrine disease $[42,46,53,54]$ and elevated serum prolactin (PRL) $[31-33,35,37,38$, $41,44,46-48,50-52]$.

\section{Interventions}

Seven nutritional supplements and four herbal medicines were investigated (Table 1).The treatment durations ranged from thirty days [39] to seven months [49] with most treatment durations (13 studies) of eight to twelve weeks (Table 1). Inositol was investigated in eight studies and three investigated omega three fish oils. Calcium plus vitamin $\mathrm{D}$, vitamin $\mathrm{D}$ alone, selenium and Cinnamomum $s p$. were investigated in two studies and nutritional supplements B complex vitamins, chromium, and herbal medicines Camellia sinensis, Mentha spicata and Cimicifuga racemosa were investigated in single studies. Calcium plus vitamin D, B complex, inositol,

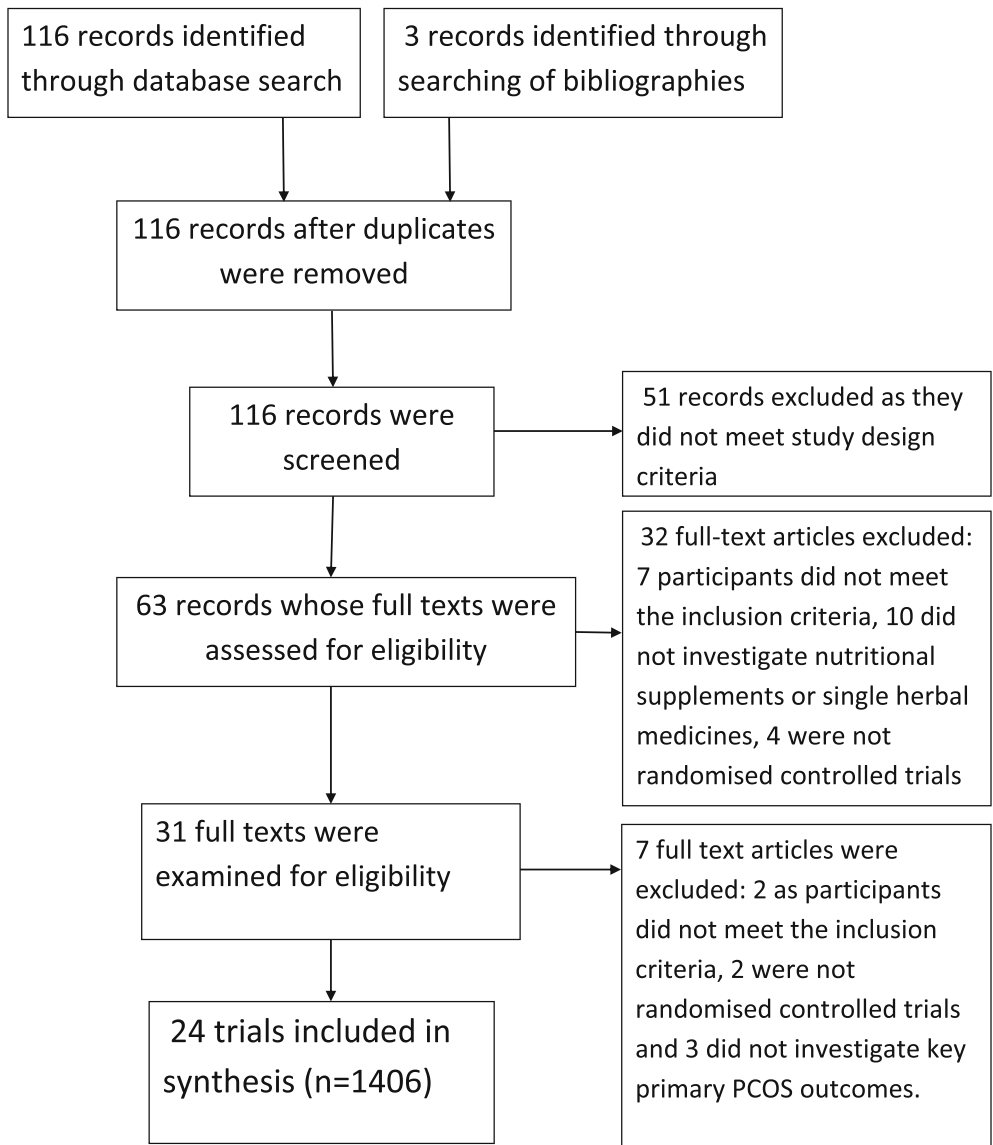

Fig. 1 PRISMA (flow chart) diagram 
Table 1 Interventions: Nutritional supplements and herbal medicine

\begin{tabular}{|c|c|c|c|c|}
\hline & Reference & Dose & Control & Duration \\
\hline \multirow[t]{3}{*}{$\begin{array}{l}\text { Omega } 3 \text { fish } \\
\text { oils }\end{array}$} & $\begin{array}{l}\text { Cussons A J; Watts GF; Mori } \\
\text { TA; Stuckey BGA. } 2009 \text { [34] }\end{array}$ & $\begin{array}{l}4000 \text { mg per day EPA } 675 \text { mg; DHA } \\
1400 \text { mg } \\
\text { (Ocean nutrition }{ }^{\circledast} \text { by Halifax, Nova } \\
\text { Scotia, Canada) }\end{array}$ & $\begin{array}{l}\text { Placebo Olive oil } 4000 \text { mg } \\
\text { (Cardinal Health Australia, Vic Australia) }\end{array}$ & 8 weeks \\
\hline & $\begin{array}{l}\text { Mohammadi E; Rafraf M; } \\
\text { Farzadi L; Asghari-Jafarabadi M; } \\
\text { Sabour S. } 2012 \text { [43] }\end{array}$ & $\begin{array}{l}4000 \text { mg omega } 3 \text { (EPA } 720 \text { mg; DHA } \\
480 \text { mg) } \\
\text { (Good Health Company, USA) }\end{array}$ & $\begin{array}{l}\text { Placebo liquid paraffin } 2000 \text { mg in four } \\
\text { capsules } \\
\text { (Zihravi Pharma, Iran) }\end{array}$ & 8 weeks \\
\hline & $\begin{array}{l}\text { Vargas LM; Almario RU; Buchan } \\
\text { W; Kim, K; Karakas SE. } 2011 \text { [50] }\end{array}$ & $\begin{array}{l}3500 \text { mg (EPA: } 2450 \text { mg; DHA: } \\
1210 \text { mg) } \\
\text { (Nordic Naturals Watsonville, California) }\end{array}$ & $\begin{array}{l}\text { Placebo (soybean oil } 3500 \text { mg) } \\
\text { (Nordic Naturals, Watsonville California) }\end{array}$ & 6 weeks \\
\hline Chromium & $\begin{array}{l}\text { Lucidi SR; Thyer AC; Easton CA; } \\
\text { Holden AEC; Schenken RS; } \\
\text { Brzyski RG. } 2009 \text { [42] }\end{array}$ & $\begin{array}{l}\text { Chromium picolinate } 200 \mathrm{mcg} \text { per day } \\
\text { (manufacturer not reported) }\end{array}$ & $\begin{array}{l}\text { Placebo } \\
\text { (type or manufacturer was not } \\
\text { reported) }\end{array}$ & 16 weeks \\
\hline \multirow[t]{2}{*}{ Selenium } & $\begin{array}{l}\text { Hosseinzadeh F; Hosseinzadeh- } \\
\text { Attar M; Yekaninejad J; Saeed } \\
\text { M; Rashidi B. } 2016 \text { [39] }\end{array}$ & $\begin{array}{l}\text { Selenium } 200 \mathrm{mcg} \text { per day } \\
\text { (Nature Made Pharmaceutical Company, } \\
\text { California USA) }\end{array}$ & $\begin{array}{l}\text { Placebo (type not specified) } \\
\text { (Roshd Pharmaceutical Incubation } \\
\text { Centre, Tehran University, Iran) }\end{array}$ & 12 weeks \\
\hline & $\begin{array}{l}\text { Razavi M; Jamilian M; Kashan } \\
\text { Z; Fakhrieh HZ; } \\
\text { Mohseni M; Ghandi Y; } \\
\text { Bagherian T; Asemi Z } 2015 \text { [47] }\end{array}$ & $\begin{array}{l}\text { Selenium 200mcg plus Metformin } \\
1500 \text { mg per day } \\
\text { (Nature Made Pharmaceutical Company, } \\
\text { California USA) }\end{array}$ & $\begin{array}{l}\text { Placebo (cellulose) plus Metformin } \\
1500 \text { mg per day(Barij Essence } \\
\text { Pharmaceutical Company, Iran) }\end{array}$ & 8 weeks \\
\hline \multirow[t]{2}{*}{ Vitamin D } & $\begin{array}{l}\text { Ardibilli HR; Gargari BG; } \\
\text { Farzadi L. } 2012 \text { [31] }\end{array}$ & $\begin{array}{l}\text { Vitamin D. Three oral treatments of } \\
50,000 \text { IU per } 20 \text { days (D-vitin }{ }^{\circledast} \text { by } \\
\text { Zahravi Pharma Company, Iran) }\end{array}$ & $\begin{array}{l}\text { Placebo, One tablet every } 20 \text { days } \\
\text { (Zihravi Pharma Company, Iran) }\end{array}$ & 2 months \\
\hline & $\begin{array}{l}\text { Raja-Khan N; Shah J; Stetter } \\
\text { CM; Lott MEJ; Kunselman AR; } \\
\text { Dodson WC; Legro RS. [45] }\end{array}$ & $\begin{array}{l}\text { Vitamin D } 12000 \text { IU per } \\
\text { day(Cholecalciferol in soy lecithin) } \\
\text { (Maximum D3 }{ }^{\oplus} \text { by BTR Group, USA) }\end{array}$ & $\begin{array}{l}\text { Placebo per day (soy lecithin without } \\
\text { cholecalciferol) (BTR Group, USA) }\end{array}$ & 12 weeks \\
\hline \multirow[t]{2}{*}{$\begin{array}{l}\text { Vitamin D plus } \\
\text { Calcium }\end{array}$} & $\begin{array}{l}\text { Rashidi R; Haghollahi F; } \\
\text { Shariat M; Zayerii F. } 2009 \text { [46] }\end{array}$ & $\begin{array}{l}\text { Calcium } 1000 \text { mg + vitamin D 400mcg } \\
\text { per day } \\
\text { (Cal D tablets by Tehran Derou, Iran) }\end{array}$ & $\begin{array}{l}\text { Metformin } 500 \text { mg, three times per day } \\
\text { (Minoo Darou, Iran) }\end{array}$ & 12 weeks \\
\hline & $\begin{array}{l}\text { Tehrani, HG; Mostajeran F; } \\
\text { Shahsavari S. } 2014 \text { [49] }\end{array}$ & $\begin{array}{l}\text { Calcium } 1000 \text { mg (Osvah } \\
\text { Pharmaceuticals) } \\
\text { Vitamin D 50,000 IU per fortnight } \\
\text { (Zahravi Pharmaceutical company) }\end{array}$ & $\begin{array}{l}\text { Metformin } \\
\text { (Sobhan darou pharmaceutical } \\
\text { company) }\end{array}$ & 16 weeks \\
\hline $\begin{array}{l}\text { Vitamin B } \\
\text { complex }\end{array}$ & $\begin{array}{l}\text { Kilicdag EB; Bagis T; Tarim E; } \\
\text { Aslan E; Erkanli S; Simsek E; } \\
\text { Haydardedeoglu B; and Kuscu } \\
\text { E. } 2005 \text { [52] }\end{array}$ & $\begin{array}{l}\text { B1: } 500 \text { mg; B6: } 500 \text { mg; B12: } 2000 \mathrm{mcg} \\
\text { + Metformin } 1700 \text { mg/day, (Apikobal } \\
\text { by Santa Farma Turkey) (Glugophage } \\
\text { by Merke, Turkey) }\end{array}$ & $\begin{array}{l}\text { Metformin } 1700 \text { mg/day } \\
\text { (Glucophage }{ }^{\circledast} \text { by Merke Turkey) }\end{array}$ & 12 weeks \\
\hline \multirow[t]{7}{*}{$\begin{array}{l}\text { Inositol } \\
\text { (vitamin B8) }\end{array}$} & $\begin{array}{l}\text { Artini PG; Di Berardino OM; } \\
\text { Papini F; Genazzani AD; Simi G; } \\
\text { Ruggiero M; Cela V. } 2013 \text { [30] }\end{array}$ & $\begin{array}{l}\text { Inositol } 2 \mathrm{~g} \text { and folic acid } 200 \text { mg plus } \\
\text { folic acid } 200 \mathrm{mcg} \\
\text { (Inofert }{ }^{\oplus} \text { by Ital Pharmaco, Milano, Italy) }\end{array}$ & $\begin{array}{l}\text { Folic acid } 400 \mathrm{mcg} \text { daily } \\
\text { (Inofert }{ }^{\oplus} \text { by Ital Pharmaco, Milano, Italy) }\end{array}$ & 12 weeks \\
\hline & $\begin{array}{l}\text { Costantino D: Minozzi G; } \\
\text { Minozzi F; Guaraldi C. } 2009 \text { [33] }\end{array}$ & $\begin{array}{l}\text { Inositol } 4 \mathrm{~g}+\text { folic acid 400mcg } \\
\left.\text { (Inofolic }{ }^{\oplus}\right)\end{array}$ & $\begin{array}{l}\text { Folic acid } 400 \mathrm{mcg} \\
\left.\text { (Fertifol }^{\oplus}\right)\end{array}$ & $12-16$ weeks \\
\hline & $\begin{array}{l}\text { Dona G; Sabbadin C; Fiore C; } \\
\text { Bragadin M; Giorgino FL; } \\
\text { Ragazzi E; Clari G; Bordin L; } \\
\text { Armanini D. } 2012 \text { [35] }\end{array}$ & $\begin{array}{l}\text { Inositol } 1200 \text { mg/day as powder, } \\
\text { pre-dosed presented in sachets } \\
\text { dissolved in water (manufacturer } \\
\text { not reported) }\end{array}$ & $\begin{array}{l}\text { Placebo } \\
\text { (matched powder type or manufacturer } \\
\text { not reported) }\end{array}$ & 12 weeks \\
\hline & $\begin{array}{l}\text { Gerli M; Mignosa; Di Renzo GC. } \\
2003 \text { [36] }\end{array}$ & $\begin{array}{l}\text { Inositol } 200 \text { mg } \\
\text { (Gestosan² LO.LI. Pharma, Rome, Italy) }\end{array}$ & $\begin{array}{l}\text { Placebo matched to Gestosan }{ }^{\oplus} \text {. } \\
\text { (Type or manufacturer not reported) }\end{array}$ & 20 weeks \\
\hline & $\begin{array}{l}\text { Gerli E; Papaleo A; Ferrari GC; } \\
\text { Di Renzo GC. } 2007 \text { [37] }\end{array}$ & $\begin{array}{l}\text { Inositol } 4 \mathrm{~g} \text { plus folic acid } 400 \mathrm{mcg} \\
\text { (Inofolic }{ }^{\circ} \text { O.LI. Pharma, Rome, Italy) }\end{array}$ & $\begin{array}{l}\text { Folic acid } 400 \mathrm{mcg} \\
\text { (manufacturer not specified) }\end{array}$ & 20 weeks \\
\hline & $\begin{array}{l}\text { luorno MJ; Jakubowicz DJ; } \\
\text { Baillargeon JP; Dillon P; Gunn } \\
\text { RD; Allan G; Nestler JE } 2002 \text { [40] }\end{array}$ & $\begin{array}{l}\text { Inositol (chiro) } 600 \text { mg } \\
\text { (INS-1 } 1^{\oplus} \text { by Insmed pharmaceuticals, } \\
\text { Richmond, Virginia, USA) }\end{array}$ & $\begin{array}{l}\text { Placebo } \\
\text { (type or manufacturer not reported) }\end{array}$ & 6-8 weeks \\
\hline & $\begin{array}{l}\text { Jamilian M; Farhat P; } \\
\text { Foroozanfard F; Ebrahimi FA; }\end{array}$ & $\begin{array}{l}\text { Inositol } 4 \mathrm{~g}+\text { folic acid } 400 \mathrm{mcg} \\
\text { (LO.LI. Rome, Italy) }\end{array}$ & $\begin{array}{l}\text { Metformin } \\
\text { (Tehran Darou Pharma. Tehran, Iran) }\end{array}$ & 12 weeks \\
\hline
\end{tabular}


Table 1 Interventions: Nutritional supplements and herbal medicine (Continued)

\begin{tabular}{|c|c|c|c|c|}
\hline & Reference & Dose & Control & Duration \\
\hline & $\begin{array}{l}\text { Nestler JE; JakubowczDJ; } \\
\text { Reamer P; Gunn R; Allan G. } \\
1999 \text { [44] }\end{array}$ & $\begin{array}{l}\text { Inositol } 1200 \text { mg labelled according to } \\
\text { subject number } \\
\text { (INS-1 }{ }^{\oplus} \text { by Insmed pharmaceuticals, } \\
\text { Virginia, USA) }\end{array}$ & $\begin{array}{l}\text { Placebo Labelled and packaged at } \\
\text { the same time as inositol. Type not } \\
\text { reported } \\
\text { (Insmed pharmaceuticals USA) }\end{array}$ & $6-8$ weeks \\
\hline Camellia sinensis & $\begin{array}{l}\text { Chan CW; Marcel Koo MWL; } \\
\text { Ng EHY; Tang OS; Yeung WSB, } \\
\text { Ho P-C. } 2006 \text { [32] }\end{array}$ & $\begin{array}{l}\text { Camellia sinensis tea infused in boiled } \\
\text { water for } 30 \text { mins, freeze dried and } \\
\text { encapsulated. Epigallocatechin (EGCG) } \\
\text { standardised to } 373.92 \mathrm{mg} \pm 20.57, \\
\text { equivalent to } 1.5 \text { cups of tea. }\end{array}$ & $\begin{array}{l}\text { Placebo capsules } \\
\text { Identical in appearance. Type or } \\
\text { manufacturer not specified. }\end{array}$ & 12 weeks \\
\hline $\begin{array}{l}\text { Cimicifuga } \\
\text { racemosa }\end{array}$ & $\begin{array}{l}\text { Shahin AY; Mohammed SA. } \\
2014 \text { [48] }\end{array}$ & $\begin{array}{l}120 \text { mg Cimicifuga extract plus } \\
\text { clomiphene } 150 \text { mg on menstrual cycle } \\
\text { days } 3-7 \text {, trigger injection ( } 10,000 \text { IU, } \\
\text { intramuscular Human Chorionic } \\
\text { Gonadotropin) and progesterone } \\
\text { supplementation (50mcg per day for } \\
\text { two days following trigger injection) } \\
\text { (Klimadynon }{ }^{\oplus} \text { Bionorica, Neumarkt, } \\
\text { Germany) } \\
\text { (Pregnyl, Organon, Holland, The } \\
\text { Netherlands) }\end{array}$ & $\begin{array}{l}\text { Clomiphene, } 150 \text { mg per day } \\
\text { administered on menstrual cycle } \\
\text { days three to seven, a trigger injection } \\
\text { (HCG) and progesterone support } \\
\text { (50mcg per day for two days following } \\
\text { trigger injection). } \\
\text { (Pregnyl, Organon, Holland, The } \\
\text { Netherlands) }\end{array}$ & $\begin{array}{l}\text { Three } \\
\text { alternate } \\
\text { menstrual } \\
\text { cycles }\end{array}$ \\
\hline \multirow[t]{2}{*}{ Cinnamomum sp. } & $\begin{array}{l}\text { Wang JG; Anderson RA; } \\
\text { Graham GM; Chu MC } \\
2007 \text { [51] }\end{array}$ & $\begin{array}{l}\text { Cinnamomum sp. } 1000 \text { mg per day } \\
\text { (Integrity Nutriceuticals, Sarasota, } \\
\text { Florida USA) }\end{array}$ & $\begin{array}{l}\text { Placebo } \\
\text { (Type or manufacturer were not } \\
\text { reported) }\end{array}$ & 8 weeks \\
\hline & Kort DH; Lobo RA. 2014 [41] & $\begin{array}{l}\text { Cinnamomum sp. } 1500 \text { mg per day } \\
\text { (Cinnulin PF Integrity Nutraceuticals } \\
\text { International, USA) }\end{array}$ & $\begin{array}{l}\text { Placebo (identical) } \\
\text { (Integrity Nutraceuticals International) }\end{array}$ & 6 months \\
\hline Mentha spicata & Grant, P. 2009 [38] & $\begin{array}{l}\text { Tea bags standardised content of dried } \\
\text { Mentha spicata leaves. Uniform } \\
\text { instructions for preparation provided to } \\
\text { participants }\end{array}$ & $\begin{array}{l}\text { Chamomile tea uniform preparation } \\
\text { instructions provided. }\end{array}$ & 30 days \\
\hline
\end{tabular}

selenium and Cimicifuga racemosa were compared with pharmaceutical controls (metformin and clomiphene citrate) $[47-50,53,54]$.

\section{Outcomes}

Four studies reported on the primary outcome, menstrual regularity $[33,42,47,50]$. Seven trials reported on adverse effects (164 women) [41-43, 45, 46, 52, 53]. Seven studies reported on secondary menstrual cycle characteristics [34, $37,38,41,45,49,50]$. One trial reported on polycystic ovaries [49]. Three studies reported on pregnancy rates [47-49] and one on miscarriage [49]. Fourteen trials reported on the outcomes of biochemical hyperandrogenism [31, 33-36, 39-43, 45, 48, 51, 54]. Four trials reported on the outcomes of clinical hyperandrogenism with validated tools including the modified Ferriman Gallwey score to measure degree of hirsutism [39, 48, 50, 54], and acne with the dermatology quality of life index for health related quality of life [39]. Eight trials reported anthropometric outcomes of participants [33, 37, 38, 44, 50, 52-54]. Eighteen trials reported metabolic biochemistry including fasting glucose and insulin [31-38, 40-46, 51-53].

\section{Risk of bias for included studies}

All 24 studies were assessed as having some risk of bias (Fig. 2). Incomplete or poor reporting by most trial authors impacted on the assessment, and consequently an unclear risk of bias for at least two characteristics was determined in 23 studies [31-52, 54]. Seven studies were found to have high risks of bias in at least three domains including performance [49-51, 54], detection $[49,50,54]$, attrition $[37,38,51,53]$, reporting [37, 38, $50,53]$ and other forms of bias [37, 38, 49-51, 53, 54]. Fourteen studies were at high risk for other sources of bias including nine with imbalanced baseline characteristics [31, 33, 37, 38, 40, 41, 43-45, 54], insufficient time to detect change [32, 34, 39], and an active control reported as placebo (placebo consisted of lecithin which is a natural source of inositol [55]) [17]. Three studies did not report the effect of high attrition (over 30\%) on baseline menstrual characteristics in the test and control groups [37, 38, 42]. Twelve studies declared conflicts of interest $[31,32,35,36,42,44-46,48,49,51,54]$. Ten studies reported no conflicts of interest and two studies reported potential conflicts of interest due to authors' affiliations [46;45]. Fourteen studies reported sources of study funding [32, 35, 36, 40-49, 51]. Three studies were funded with grants from the pharmaceutical industry [35, 41, 45], twelve studies were funded by university or research institute grants [32, 36, 40, 42-49, 51] and one study was funded by both pharmaceutical industry and an institute grant [45]. 


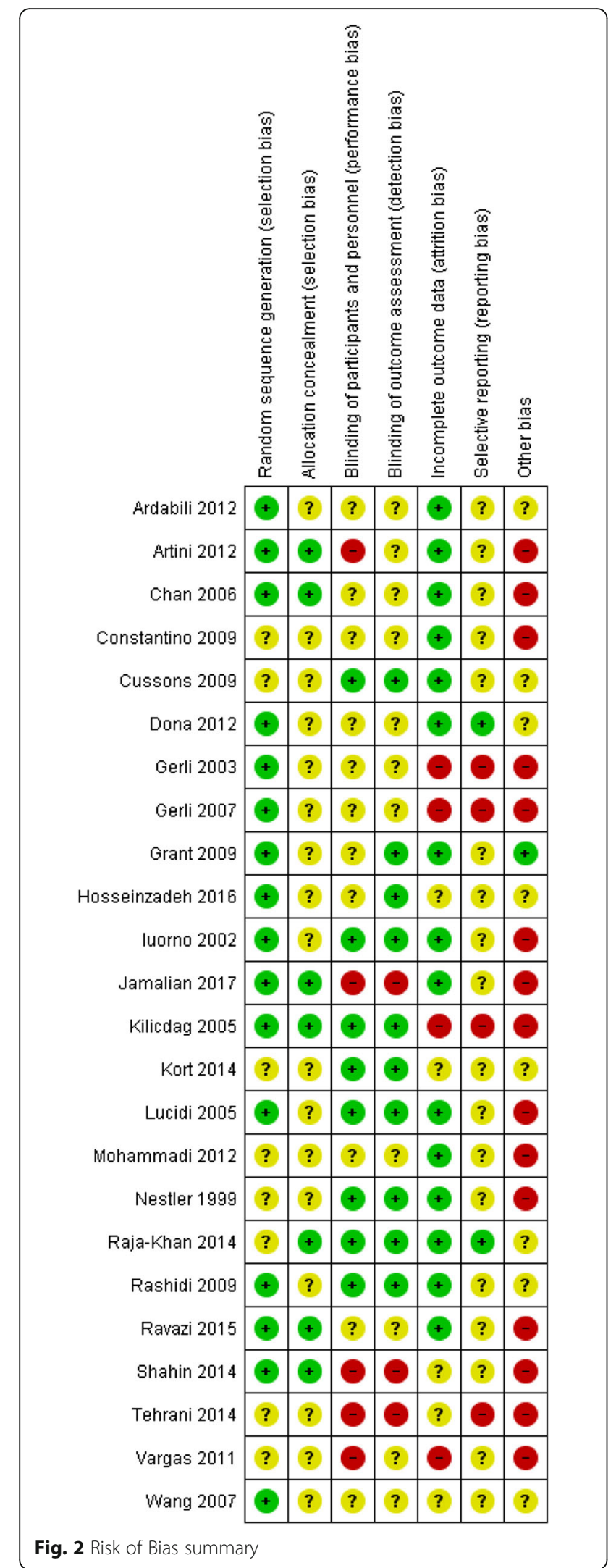

\section{Effects of interventions}

Data from two trials were not included in analyses. One trial used a cross-over trial design and did not report first phase outcomes [35]; the other study reported outcomes as the median rather than the mean [33]. Where data could not be included in a meta-analysis a narrative reporting of the findings was presented.

\section{Nutritional supplements for PCOS}

Calcium plus vitamin D Two trials (78 women) compared calcium plus vitamin D with metformin for the number of women with regular menstrual cycles (21-35 days) $[47,50]$. There was no evidence of an improved effect (RR $0.66,95 \%$ CI 0.35 to $\left.1.23, p=0.19, \mathrm{I}^{2} 0 \%\right)$. Adverse effects were not reported.

\section{Secondary outcomes}

Rashidi and colleagues reported on the number ovarian cysts defined as immature follicles (diameter 5-9 $\mathrm{mm}$ ) and pregnancy rates for calcium plus vitamin $\mathrm{D}$ against metformin [47]. No difference between groups was found. Tehrani and colleagues reported on BMI and hirsutism (defined as Ferriman Gallwey score over 8) [50]; no difference was found between groups.

Vitamin B complex One study investigated B complex vitamins plus metformin compared with metformin alone, for 32 women [53]. Results were reported as 'women taking Metformin' and between group differences were not reported. No data were available for B complex vitamins on menstrual regularity. No difference for adverse events was found.

\section{Secondary outcomes}

Four secondary outcomes for vitamin B complex plus metformin were reported. No significant effects were found for pregnancy rates, waist to hip ratio or HOMA-IR.

Inositol (vitamin B8) No trials reported on the primary outcome menstrual regularity for inositol (Table 2). Two trials (64 women) reported on adverse events for inositol [41, 45]. No adverse effects were found for inositol against placebo $[41,45]$.

\section{Secondary outcomes}

Significant benefits were found for inositol versus placebo and active controls (90 women) for the secondary outcomes of reproductive characteristics and hyperandrogenism (Fig. 3) and (Table 2).

The number of ovulations was significantly higher for women taking inositol (two studies, 64 women, RR 3.13, 95\% CI 1.68 to $5.83, p<0.001$ ). Two trials reported on a subgroup of women wanting to conceive (84 women) $[37,38]$ and a third trial included women preparing to undergo medically assisted fertility treatment (50 women) [31]. Meta analyses of these three studies (134 
Table 2 Inositol for reproduction, hyperandrogenism, metabolism, anthropometry and risk factors

\begin{tabular}{|c|c|c|c|c|c|c|}
\hline & $\begin{array}{l}\text { Treatment } \mathrm{n}^{*} \\
\text { (number of } \\
\text { effects)/N }\end{array}$ & $\begin{array}{l}\text { Control } n^{*} \text { (number } \\
\text { of effects)/N }\end{array}$ & $\begin{array}{l}\text { Number of RCTs } \\
\text { Heterogeneity } \\
\left(I^{2}, p \text { value }\right)\end{array}$ & $\begin{array}{l}\text { Treatment } \\
\text { effect MD or RR }\end{array}$ & $95 \% \mathrm{Cl}$ & $p$ value \\
\hline \multicolumn{7}{|l|}{ Hyperandrogenism } \\
\hline Free testosterone $\mathrm{nmol} / \mathrm{l}$ & 55 & 51 & $\begin{array}{l}3[22,23,30] \\
0 \%, p=0.94\end{array}$ & MD -0.02 & -0.10 to 0.06 & 0.62 \\
\hline Total testosterone $\mathrm{nmol}^{* *} \mathrm{l}$ & 103 & 89 & $\begin{array}{l}5[34,36,41,45,54] \\
32 \%, p=0.0009\end{array}$ & MD 0.99 & -1.54 to -0.44 & $<0.001$ \\
\hline Androstenidione $\mathrm{nmol} / \mathrm{l}$ & 73 & 59 & $\begin{array}{l}4[22,23,30,37] \\
0 \%, p=1.0\end{array}$ & MD 3.59 & -4.62 to -2.55 & $<0.001$ \\
\hline $\begin{array}{l}\text { Sex hormone binding globulin } \\
\text { (SHBG) } n m o l / l\end{array}$ & 55 & 67 & $\begin{array}{l}2[22,23] \\
0 \%, 0.50\end{array}$ & MD 40.67 & 25.83 to 55.50 & $<0.001$ \\
\hline Modified Ferriman Gallwey score & 30 & 30 & $1[54]$ & MD 0.90 & -1.18 to 2.98 & 0.40 \\
\hline \multicolumn{7}{|l|}{ Reproduction } \\
\hline $\begin{array}{l}\text { Number of days to ovulation } \\
\text { (number of days) }\end{array}$ & 181 & 194 & $\begin{array}{l}2[24,25] \\
0 \%, p=0.90\end{array}$ & MD 17.7 & -31.4 to -4.1 & 0.01 \\
\hline $\begin{array}{l}\text { Ratio of luteal week to total trial luteal } \\
\text { weeks: total trial weeks }\end{array}$ & $166 / 697$ & $140 / 1000$ & $\begin{array}{l}2[24,25] \\
0 \%, p=0.90\end{array}$ & RR 1.7 & 1.39 to 2.08 & $<0.001$ \\
\hline Number of ovulations & $25 / 32$ & $8 / 32$ & $\begin{array}{l}2[22,23] \\
0 \%, p=0.95\end{array}$ & RR 3.13 & 1.68 to 5.83 & 0.001 \\
\hline Number of women who did not ovulate & $16 / 181$ & $34 / 194$ & $\begin{array}{l}2[24,25] \\
0 \%, p=0.95\end{array}$ & RR 0.5 & 0.29 to 0.86 & 0.01 \\
\hline Pregnancy & 18/71 & $6 / 63$ & $\begin{array}{l}3[24,25,36] \\
0 \%, p=0.95\end{array}$ & RR 2.78 & 1.19 to 6.5 & 0.02 \\
\hline Live births & $8 / 25$ & $3 / 25$ & $1[36]$ & RR 2.67 & 0.80 to 8.90 & 0.11 \\
\hline $\mathrm{FSH} \mathrm{mIU} / \mathrm{l}$ & 25 & 25 & $1[36]$ & MD 1.4 & -1.63 to -1.17 & 0.001 \\
\hline LH mIU/l & 25 & 25 & $1[36]$ & MD 3.5 & -4.9 to -2.1 & 0.001 \\
\hline FSH:LH ratio & 25 & 25 & $1[36]$ & MD 0.4 & -0.68 to -0.12 & 0.006 \\
\hline \multicolumn{7}{|l|}{ Anthropometric } \\
\hline \multirow[t]{2}{*}{$B M I^{* * *}$} & 48 & 38 & $2[37,54]$ & MD -0.30 & -1.10 to 0.50 & 0.46 \\
\hline & 57 & 57 & $\begin{array}{l}3[22,23,36] \\
1^{2} 31 \%, p=0.24\end{array}$ & MD 0.40 & -0.07 to -0.86 & 0.86 \\
\hline Waist to hip ratio & 32 & 32 & $\begin{array}{l}2[22,23] \\
0 \%, p=0.32\end{array}$ & $M D-0.02$ & -0.03 to -0.01 & 0.01 \\
\hline \multicolumn{7}{|l|}{ Metabolic hormones } \\
\hline Fasting glucose $\mathrm{mmol} / \mathrm{l}$ & 73 & 59 & $\begin{array}{l}4[22,23,30,37] \\
0 \%, P=0.52\end{array}$ & MD 1.17 & -1.72 to -0.63 & $<0.001$ \\
\hline Fasting insulin $\mu \mathrm{U} / \mathrm{ml}$ & 73 & 59 & $\begin{array}{l}4[22,23,30,37] \\
18 \%, p=0.30\end{array}$ & MD 2.7 & -3.9 to -1.5 & $<0.001$ \\
\hline \multirow[t]{2}{*}{ HOMA-IR $\mathbb{R}^{* * * *}$} & 25 & 25 & $1[36]$ & $M D-1.30$ & -1.60 to -1.00 & $<0.001$ \\
\hline & 18 & 8 & $1[37]$ & $M D-0.60$ & -0.89 to -0.30 & $<0.001$ \\
\hline \multicolumn{7}{|l|}{ Risk factors } \\
\hline Cholesterol mmol// & 32 & 32 & $\begin{array}{l}2[22,23] \\
27 \%, p=0.24\end{array}$ & MD 0.56 & -1.07 to -0.04 & 0.04 \\
\hline Triglycerides mg/dL & 32 & 32 & $\begin{array}{l}2[22,23] \\
8 \%, p=0.30\end{array}$ & MD 31.6 & -61.1 to -2.0 & 0.04 \\
\hline
\end{tabular}


Table 2 Inositol for reproduction, hyperandrogenism, metabolism, anthropometry and risk factors (Continued)

\begin{tabular}{|c|c|c|c|c|c|c|}
\hline & $\begin{array}{l}\text { Treatment } \mathrm{n}^{*} \\
\text { (number of } \\
\text { effects)/N }\end{array}$ & $\begin{array}{l}\text { Control } n^{*} \text { (number } \\
\text { of effects)/N }\end{array}$ & $\begin{array}{l}\text { Number of } R C T s \\
\text { Heterogeneity } \\
\left(I^{2}, p \text { value }\right)\end{array}$ & $\begin{array}{l}\text { Treatment } \\
\text { effect MD or RR }\end{array}$ & $95 \% \mathrm{Cl}$ & $p$ value \\
\hline $\mathrm{HDLmg} / \mathrm{dL}$ & 22 & 22 & $1[22]$ & MD 0 & -4.73 to 4.73 & 1.0 \\
\hline LDL mg/dL & 22 & 22 & $1[22]$ & $M D-2.0$ & -13.7 to 9.7 & 0.74 \\
\hline CRP & 30 & 30 & 1 & $M D-2.2$ & -3.57 to -0.83 & $<0.01$ \\
\hline
\end{tabular}

*calculated for dichotomous outcomes only

**five trials with significant heterogeneity $\left(I^{2}=75 \%, p=0.003\right)$ following analyses incorporating random effects

***five trials with significant heterogeneity $\left(I^{2}=89 \%, p<0.0001\right)$ following analyses incorporating random effects, and not combined

${ }_{* * * *}^{*}$ two trials with significant heterogeneity $\left(\mathrm{I}^{2}=91 \%, p-0.0009\right)$ following analyses incorporating random effects, and not combined

women) demonstrated pregnancy rates were significantly higher for women taking inositol compared to controls (RR 2.8, 95\% CI 1.2 to $6.5, p=0.02$ ).

Significant benefits were found for the secondary outcomes of total testosterone, (five studies, 192 women, MD $0.99 \mathrm{nmol} / \mathrm{l}, 95 \%$ CI -1.54 to $-0.44, p<0.001$ ) [34, 36, 41, 45, 54] (Fig. 3) and androstenidione (four studies, 132 women, MD $3.59 \mathrm{nmol} / \mathrm{l}, 95 \% \mathrm{CI}-4.6$ to $-2.6, p<0.001$ ) [34, 36, 41, 45] and SHBG (two studies, 122 women, MD $40.67 \mathrm{nmol} / \mathrm{l}, 25.8$ to $55.5, p<0.01$ ) [41, 45]. No significant benefit was found for free testosterone following treatment with inositol [34, 36, 41, 45] or for clinical hyperandrogenism (Ferriman Gallwey score) for inositol compared with metformin [54].

Significant benefits were found for fasting glucose (four trials, 132 participants, MD-1.17, 95\% CI -1.72 to $-0.63, p<0.01$ ), [34, 36, 41, 45]; fasting insulin (four trials, 132 participants, $\mathrm{MD}-2.7 \mu \mathrm{U} / \mathrm{ml}, 95 \% \mathrm{CI}-3.9$ to $-1.5, p<0.01)[34,36,41,45]$ and reduced insulin resistance (HOMA-IR) (two trials, 76 participants, MD -1.3, 95\% CI -1.6 to $-1.0, p<0.01$ ) [31, 36] (Table 2). Significant heterogeneity was found for the two trials investigating inositol for HOMA-IR $\left(\mathrm{I}^{2}=91 \%, p<0.001\right)$ and effects were adjusted using a random effects model. Study heterogeneity was likely due to different characteristics of participants at baseline (normal weight [36] and overweight [31]), different doses of inositol and types of controls (1200 g per day versus placebo [36] and $2 \mathrm{~g}$ plus folic acid versus folic acid alone [31]).

Significant benefits were found for the secondary outcome waist to hip ratio (two studies, 64 women, MD $-0.02,95 \%$ CI -0.03 to $-0.01, p=0.01$ ) [41, 45]. Mixed results were found for inositol with BMI in five trials [31, 36, 41, 45, 54] (Table 2). One trial showed significant improvements in BMI [36], whilst three others found no significant difference between groups [31, 45, 54]. A fifth trial found significantly increased BMI for women taking inositol compared to placebo controls however this study included lean participants and mean BMI at endpoint remained within normal BMI range $(22.3 \pm 0.3)$ [41]. Significant heterogeneity of trials prevented meta-analysis $\left(\mathrm{I}^{2}=89 \%, p<0.00001\right)$. The high heterogeneity may be explained clinically with variable baseline BMI categories of participants ranging from normal [36, 41, 54], overweight [31, 54] and obese [45]. Dose variation of inositol may have also contributed to study heterogeneity with daily doses of $600 \mathrm{mg}$ [41], $1200 \mathrm{mg}[36,45]$ and $2 \mathrm{~g} \mathrm{[31]} \mathrm{[36])} \mathrm{and} \mathrm{durations} \mathrm{of} \mathrm{treat-}$ ment for six [41, 45] and twelve weeks [31, 36, 54]) [54]. Significant benefits were found for total cholesterol (two 
trials, 64 women, MD $0.56 \mathrm{mmol} / \mathrm{l}, 95 \% \mathrm{CI}-1.07$ to -0.04 , $p=0.04$ ) $[41,45]$ and triglycerides (two trials, 64 women, MD 31.6 mg.dl, 95\% CI -61.1 to $-2.0, p=0.04)[41,45]$ following treatment with inositol compared to placebo controls. Reduced inflammation (c-reactive protein (CRP)) was found for inositol compared to metformin in one study (MD $-2.2,95 \%$ CI -3.57 to $-0.83, p<0.01$ ) [54].

Chromium supplements One small study reported on chromium compared to placebo in ten women with PCOS [43]. The study did not report on menstrual regulation. No adverse events were reported for either chromium or placebo. Five secondary outcomes free testosterone, total testosterone, ovulation, FSH and LH were reported. No significant treatment effects were found for any outcomes.

Vitamin D Two RCTs examined vitamin D supplements compared with a placebo in 78 women with PCOS [32, 46]. Heterogeneity of studies was not significant $\left(\mathrm{I}^{2}=0 \%\right)$ and data were pooled for analyses. The effects of vitamin $\mathrm{D}$ on menstrual regulation were not reported. No adverse effects were found in one study [46].

Secondary outcomes

No significant treatment effect was found for vitamin D compared with placebo for metabolic hormones including fasting glucose, fasting insulin, insulin resistance or insulin sensitivity [32, 46]. One study including 28 women found no evidence of improvements for vitamin D compared to placebo for free and total testosterone, cholesterol and high and low density lipoproteins triglycerides and inflammation (CRP) [46].

Omega three fish oils Two studies investigated omega 3 supplements against placebo in 95 women [44, 51]. Neither study reported on menstrual regulation or adverse effects.

\section{Secondary outcomes}

A significant effect was found for the risk marker total cholesterol in women receiving omega 3 fish oils compared to placebo controls (two trials, 95 women,
-0.49 mmol, $95 \%$ CI -0.62 to $-0.35, p<0.001)[44,51]$ (Fig. 4).

No evidence of an effect was found for free testosterone or SHBG [51], waist to hip ratio [44] or for inflammation (CRP) [44]. Findings were mixed for some secondary outcomes including triglycerides, HDL and LDL [44, 51], BMI [44, 51], fasting glucose and insulin resistance $[44,51]$. Clinical heterogeneity was likely due to the dose of omega three. One trial received treatment of $3.5 \mathrm{~g}$ (EPA $258 \mathrm{mg}$ and DHA $242 \mathrm{mg}$ ) for six weeks [51], which was less than the other trial where women received $4 \mathrm{~g}$ (EPA $720 \mathrm{mg}$ and DHA $480 \mathrm{mg}$ ) over eight weeks and additionally due to wide variation in mean BMI at baseline. Both trials included women with a BMI that ranged between 25 and 40-45 however the mean BMI for women in one trial was in the overweight category (BMI 28) [51] compared to morbidly obese (BMI 36) in the other [44]. Significant effects were found for morbidly obese women taking the higher dose of fish oils [44] for fasting glucose $(-0.33 \mathrm{mmol} / \mathrm{l}, 95 \% \mathrm{CI}-0.26$ to $-0.40, p<0.001)$, insulin resistance (HOMA-IR) $(-1.23$, $95 \%$ CI -1.6 to $-0.86, p<0.001)$ and reduced LDLs $(-0.88$, $95 \%$ CI -1.04 to $-0.72, p<0.01)$ [44].

Selenium Two studies investigated selenium supplements against placebo in 109 women [40, 48]. One investigated added metformin in both study groups [48]. Neither menstrual regulation nor adverse effects were investigated in either study.

\section{Secondary outcomes}

A significant effect was found in one study for selenium against placebo in 53 women for fasting glucose (MD $4.66 \mathrm{mg} / \mathrm{dl}, 95 \% \mathrm{CI} 3.23$ to $6.09, p<0.00001$ ), insulin resistance (HOMA-IR) (MD $0.2495 \% \mathrm{CI} 0.06$ to 0.42 , $p=0.008$ ), total testosterone (MD $0.18 \mathrm{ng} / \mathrm{ml}, 95 \% \mathrm{CI}$ 0.09 to $0.27, p<0.0001$ ), SHBG (MD $12.80 \mathrm{nmol} / \mathrm{l}, 95 \%$ CI 6.22 to $19.38, p<0.0001)$ and FAI (MD 3.93, 95\% CI 3.54 to $4.32, p<0.00001$ ) [40]. No effect was found for fasting insulin. Ravazi and colleagues [48] examined selenium in addition to pharmaceutical management (metformin) against metformin plus placebo in 64

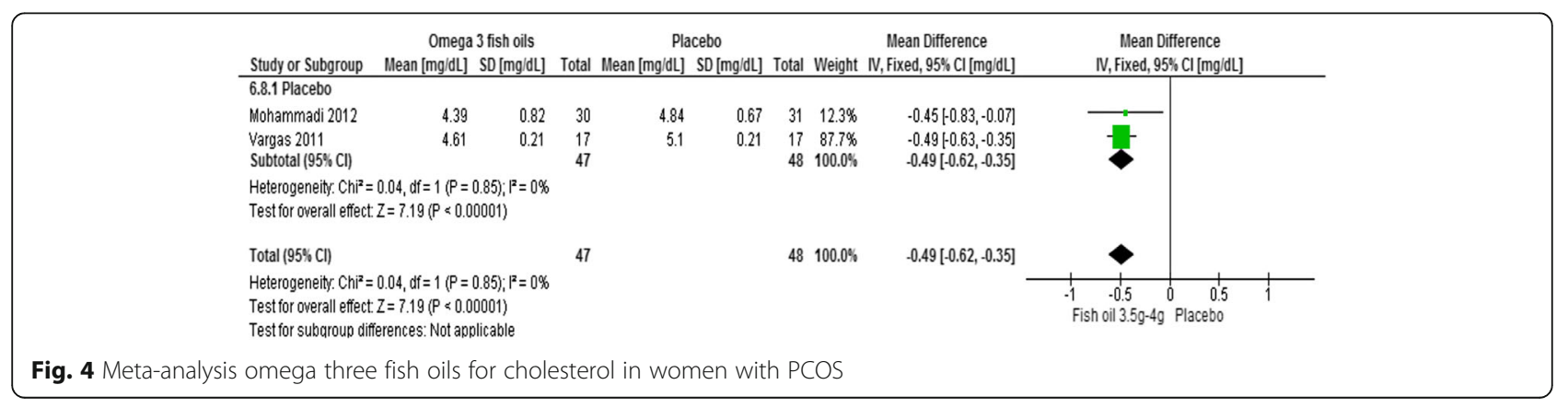


women. No evidence of an additive effect was found for free testosterone, inflammation or pregnancy rates.

Herbal medicines for PCOS One trial reported on Camellia sinensis (green tea) capsules compared with placebo for menstrual regularity [33]. Chan and colleagues (2006) investigated the proportion of amenorrheic women over a three month period (34 women) [33]. No difference was found between groups. Adverse effects were not investigated.

Secondary outcomes

Chan and colleagues (2006) reported on secondary outcomes however data were presented as median differences and the interquartile range and analysed for changes over time. Consequently data were not suitable for inclusion in analyses in this review [33]. No significant differences in median values over time were found for women taking Camellia sinensis or placebo for testosterone, SHBG, FAI, androstenidione, FSH, LH, BMI, waist to hip ratio, insulin, glucose, cholesterol, triglycerides HDL or LDL.

Cimicifuga Racemosa One study examined the combination of Cimicifuga racemosa and the pharmaceutical agent Clomiphene citrate (clomiphene) against clomiphene alone in 194 women [49]. Menstrual regulation or adverse effects were not reported.

Secondary outcomes

A significant treatment effect was found for the combination group for reduced days to ovulation (MD -3 days, $95 \%$ CI -3.51 to $-2.49, p<0.01$ ), pregnancy rates (RR 1.98, 95\% CI 1.19 to 3.31, $p<0.01$ ), FSHmIU/l (MD 0.40, 95\% CI 0.22 to $0.58, p<0.01$ ), LH mIU/l (MD $-2.3,95 \%$ CI -2.55 to $-2.05, p<0.01)$, and reduced miscarriage (RR $0.62,95 \%$ CI 0.22 to $1.74, p=0.36$ ).

Cinnamomum sp. No evidence of a menstrual regulation effect was found in one study investigating Cinnamomum sp. compared with placebo in 17 women [42]. Four nonserious adverse events were reported for Cinnamomum $s p$. [42]. These included headache, heartburn, menstrual cramps, nausea and diarrhoea. No adverse events were reported in the second study by Wang and colleagues [52]. Use of a variable dose may explain the different findings for adverse effects; one investigated $1500 \mathrm{mg}$ of Cinnamomum $s p$. per day over six months [42] and the other $1000 \mathrm{mg}$ per day over eight weeks [52].

\section{Secondary outcomes}

Two studies reported on metabolic hormones following treatment with Cinnamomum sp. compared to placebo $[42,52]$. Primary data were not reported by Wang and colleagues and between groups could not be examined [52]. No evidence of a treatment effect for Cinnamomum $s p$. was found for insulin resistance (HOMA-IR) or insulin sensitivity (QUICKI) [42].

Mentha Spicata No data on primary outcomes were reported.

\section{Secondary outcomes}

A significant treatment effect was found for Mentha spicata compared to Matricaria recutita (chamomile) tea for reduction in total testosterone in 41 women (MD $0.18 \mathrm{nmol} / \mathrm{l}, 95 \% \mathrm{CI}-0.34$ to $-0.02, p=0.03$ ) [39]. No significant treatment effects were found for free testosterone, hirsutism or the dermatology index quality of life assessment.

\section{Discussion}

This systematic review included 24 randomised controlled trials of 1406 women with PCOS. Seven nutritional supplements and four herbal medicines were investigated and meta-analyses were reported for calcium plus vitamin D, Cinnamomum sp., inositol (vitamin B8), omega three fish oils and vitamin D. Menstrual regularity, a defining feature of critical interest to women with PCOS [25], was examined in only four studies and no significant treatment effect compared to controls was found. However secondary outcomes of time to ovulation, ovulation rates, hyperandrogenism, reproductive and metabolic hormones, waist to hip ratio, cholesterol and triglycerides were significantly improved by inositol and total cholesterol was significantly lowered by omega three fish oils compared to controls. Adverse effects were reported in seven studies for four nutritional supplements and one herbal medicine. Mild adverse effects were found for Cinnamomum $s p$. There were no long term investigations of safety.

Findings for the primary outcome, menstrual regulation This review found no single nutritional supplement or herbal medicine significantly improved the primary outcome, menstrual regulation. The oral contraceptive pill (OCP) is first line pharmaceutical treatment for menstrual regularity in women with PCOS [9], however it is contraindicated in women with increased cardiovascular risks $[12,15]$ and women with PCOS have reported preferences for effective alternatives [15]. Metformin is an effective alternative however women with PCOS have reported high rates of unpleasant side effects [13].

There is low quality evidence from RCTs for menstrual regulation included in this review. Trials were characterized by a high risk of selection, performance and detection biases and an unclear risk of reporting bias. Evidence from methodologically sound RCTs is needed to examine the reliability of findings for calcium plus vitamin D compared with metformin for menstrual regulation. 
Chan (2006) and colleagues found a treatment effect on the primary outcome in a sub-analysis investigating Camellia sinensis with a lower proportion of amenorrheic women at twelve weeks for women taking Camellia sinensis compared to placebo controls, however differences between groups were not significant [33]. Findings were limited by the small sub-group sample size (only 14 women), the study was underpowered for the number of amenorrheic women and was assessed as low quality due to not reporting baseline characteristics of subgroups and for reporting on medians rather than means.

The findings of this review contribute to the evidence for Chinese Herbal Medicine (CHM) for oligomenorrhoea and amenorrhoea in women with PCOS [23]. Traditional Chinese herbal formulations are another form of ingestible complementary medicine of interest to women with PCOS [16]. In that systematic review which included Chinese and English language papers, only one small, low quality study investigated menstrual cyclicity outcomes for CHM and found an improved menstrual regulation response from the oral contraceptive pill (Diane 35) when combined with CHM compared to Diane 35 alone (RR 2.6, 95\% CI 1.06 to 6.39, $p=0.04)$. We have some low grade evidence to add to women's treatment decisions for menstrual regularity; calcium plus vitamin D, compared to metformin does not appear to provide any further benefits on this outcome. We agree that the evidence for ingestible CMs for menstrual regulation is poor quality and inconclusive due to the absence of findings from methodologically sound clinical studies.

\section{Findings for the nutritional supplement inositol}

This review provides some support for a therapeutic role of inositol. As an essential structural component of cell membranes, inositol is needed for cell membrane signalling functions including those involving neurological signalling in the brain, second messenger systems and insulin signal transduction. Impaired insulin signalling in skeletal muscle and adipose tissue has a pathogenic role in conditions of insulin resistance $[56,57]$. It has been proposed that ovarian theca cells of women with PCOS remain sensitive to insulin despite insulin resistance, and theca cell androgen production continues through an alternative insulin signal transduction system [58]. Evidence for a range of doses of inositol suggests therapeutic supplementation may improve metabolic profile in women with PCOS in addition to reduced biochemical hyperandrogenism and improved ovulation and pregnancy rates. However, only one small study included in this review examined live birth rates and no significant improvements were found compared to controls. As healthy, live birth outcomes are the primary goal of all interventions for women with PCOS seeking treatment for infertility there is a need for more high quality randomised controlled trials to report on this outcome. Adverse effects of inositol were examined in only two studies. Despite the likely low risks [59] this systematic review found no long term investigations assessing safety, and the evidence of safety is not established in women with PCOS.

The findings of this review differ with the systematic review of hypoglycaemic interventions for women with PCOS [14]. Tang and colleagues found no evidence for inositol compared with placebo in two studies for improved ovulation in 327 women (Odds Ratio 5.38, 95\% CI 0.70 to $41.31, \mathrm{I}^{2} 81 \%$ ) [38, 45], or for lowered androgens, metabolic markers or blood lipids in 44 women [45]. Differences in our findings may be explained by the variable study inclusion criteria and the number of studies included in analyses. Tang and colleagues included studies only investigating the d-chiro isomer form of inositol against placebo or no treatment [14]. This present review included studies investigating any isometric form of inositol and active controls.

\section{Well-being and quality of life outcomes}

Women are likely to use nutritional supplements and herbal medicines in addition to conventional pharmaceutical management possibly to improve well-being and quality of life $[16,60]$. No studies investigated well-being or quality of life (QoL) outcomes and few studies examined safety for the use of nutritional and herbal supplements in conjunction with pharmaceutical treatment. Further evidence is needed to inform women's treatment decisions and to assess the clinical usefulness of nutritional supplements and herbal medicine for improved well-being and quality of life in women with PCOS.

\section{Limitations}

The inclusion of an English language excluded nonEnglish language research and indexations. Herbal medicine has a high prevalence of use amongst non-English speaking cultures and this review was limited by its omission of non-English language research and databases.

Other limitations of this review include the poor quality of evidence, the small number of nutritional supplements and herbal medicine interventions, the absence of QoL and well-being outcomes and lack of long term investigations of safety. Key words did not include individual nutrient and herbal possibilities or complex herbal formulations and evidence may have been missed. In addition, methodological weaknesses lead to the exclusion of many studies and the authors did not seek further information from researchers of studies where the median rather than the mean was reported. Further research using rigorous RCT design 
is needed to inform findings of this systematic review.

A narrow inclusion criteria of only RCTs that examined the three diagnostic features of PCOS were specified for this review. Further literature reviews with inclusion criteria of other clinical outcomes such as fertility, anthropometric or psychological outcomes may strengthen the findings found here and better inform clinicians and women's treatment decisions.

\section{Conclusion}

There is no high quality evidence to support the effectiveness of nutritional supplements and herbal medicine for PCOS symptoms and evidence of safety and is lacking. However for two nutritional supplements, there was some low quality evidence that suggests women with PCOS may benefit from inositol and omega three fish oil supplements. Further research is needed.

\section{Additional file}

Additional file 1: Table S1. studies excluded following full text review. (DOCX $30 \mathrm{~kb}$ )

\section{Abbreviations}

BMI: Body mass index; CHM: Chinese herbal medicine; Cl: Confidence interval; CM: Complementary medicine; CRP: C reactive protein; D3: Cholecalciferol; DHA: Docosahexaenoic Acid; EPA: Eicosapentaenoic acid; FAl: Free androgen index; FSH: Follicle stimulating hormone; HDL: High density lipoprotein; HOMA-IR: Homoeostatic model assessment of insulin resistance; IU: International units; LDL: Low density lipoprotein; LH: Luteinizing hormone; MD: Mean difference; NIH: National Institute of Health; OCP: Oral contraceptive pill; PCOS: Polycystic ovary syndrome; PRISMA: Preferred reporting items for systematic reviews and meta-analyses; PRL: Prolactin; QUICKI: Quantitative insulin sensitivity check index; RCT: Randomized control trial; RR: Relative risk; SHBG: Sex hormone binding globulin; sp.: Species
\end{abstract}

\section{Funding}

This research project was funded by Western Sydney University as doctorate candidate project funding for Susan Arentz with support from an Australian Postgraduate Award. As a medical research institute, the National Institute of Complementary Medicine receives research grants and donations from foundations, universities, government agencies and industry. Sponsors and donors provide untied funding for work to advance the vision and mission of the Institute. The project that is the subject of this article was not undertaken as part of a contractual relationship with any organisation. Authors SA and JA are also engaged in integrative medicine clinical practice.

\section{Availability of data and materials}

The datasets supporting the conclusions of this article are available on reasonable request from the author SA.

\section{Authors' contributions}

$S A, J A, C S$ and $A B$ conceived of the study and participated in its design and coordination. SA carried out the search of the literature. SA and CAS participated in study inclusion or exclusion with consensus with JA and $A B$. SA and CAS performed data extraction and JA reviewed the quality of data. $S A, C A S, J A$ and $A B$ designed and edited the tables. All authors read and approved the final manuscript.

Ethics approval and consent to participate Not applicable.
Consent for publication

Not applicable.

\section{Competing interests}

The authors declare that they have no competing interests. SA was a doctoral research student and CAS, JA and AB her supervisory panel.

\section{Publisher's Note}

Springer Nature remains neutral with regard to jurisdictional claims in published maps and institutional affiliations.

\section{Author details}

${ }^{1}$ National Institute of Complementary Medicine, Western Sydney University, Locked Bag 1797, Penrith, NSW 2751, Australia. ${ }^{2}$ School of Women's and

Children's Health, University of New South Wales, Sydney, Australia.

Received: 12 January 2017 Accepted: 16 November 2017

Published online: 25 November 2017

\section{References}

1. ESHRE. Consensus on women's health aspects of polycystic ovary syndrome (PCOS). Hum Reprod. 2012;27(1):14-24.

2. March WA, et al. The prevalence of polycystic ovary syndrome in a community sample assessed under contrasting diagnostic criteria. Hum Reprod. 2010;25(2):544-51.

3. Doherty DA, Newnham JP, Bower C, Hart R. Implications of polycystic ovary syndrome for pregnancy and for the health of offspring. Obstet Gynecol. 2015;125(6):1397-406.

4. Boomsma CM, Eijkemans MJC, Hughes EG, Visser GHA, Fauser BCJM, Macklon NS. A meta-analysis of pregnancy outcomes in women with polycystic ovary syndrome. Hum Reprod Update. 2006;12(6):673-83.

5. Dokras A. Mood and anxiety disorders in women with PCOS. Steroids. 2012:77(4):338-41.

6. Hart R, Doherty DA. The potential implications of a PCOS diagnosis on a Woman's long-term health using data linkage. J Clin Endocrinol Metab. 2014;100(3):911-9.

7. Cassar S, et al. Insulin resistance in polycystic ovary syndrome: a systematic review and meta-analysis of euglycaemic-hyperinsulinaemic clamp studies. Hum Reprod. 2016;31(11):2619-31.

8. Stepto NK, Cassar S, Joham AE, Hutchison SK, Harrison CL, Goldstein RF, Teede HJ. Women with polycystic ovary syndrome have intrinsic insulin resistance on euglycaemic-hyperinsulaemic clamp. Hum Reprod. 2013; 10 1093/humrep/des463.

9. AAPCOS. In: NHMRC, editor. Evidence-based guideline for the assessment and management of polycystic ovary syndrome. Melbourne: Jean Hailes Foundation for Women's Health on behalf of the PCOS Australian Alliance; 2011.

10. Moran LJ, Hutchison SK, Norman RJ, Teede HJ. Lifestyle changes in women with polycystic ovary syndrome. Cochrane Database Syst Rev. 2011; 10. 1002/14651858.CD007506.pub2.

11. Moran LJSR, Zoungas S, McNaughton SA, Brown WJ, Teede HJ. The contribution of diet, physical activity and sedentary behaviour to body mass index in women with and without polycystic ovary syndrome. Hum Reprod. 2013; 10.1093/humrep/det256.

12. Diamanti-Kandarakis E, Baillargeon JP, luorno MJ, Jakubowicz DJ, Nestler JE. A modern medical quandary: polycystic ovary syndrome, insulin resistance, and oral contraceptive pills. J Clin Endocrinol Metab. 2003;88(5):1927-32.

13. Legro RS, Barnhart HX, Schlaff WD, Carr BR, Diamond MP, Carson SA, Steinkampf MP, Coutifaris C, McGovern PG, Cataldo NA. Clomiphene, metformin, or both for infertility in the polycystic ovary syndrome. N Engl J Med. 2007;356(6):551-66.

14. Tang, T., J.M. Lord, R.J. Norman, E. Yasmin, A.H. Balen., Insulin-sensitising drugs (metformin, rosiglitazone, pioglitazone, D-chiro-inositol) for women with polycystic ovary syndrome, oligo amenorrhoea and subfertility. Cochrane Database Syst Rev, 2012. 5.

15. Sills ES, Perloe M, Tucker MJ, Kaplan CR, Genton MG, Schattman GL. Diagnostic and treatment characteristics of polycystic ovary syndrome: descriptive measurements of patient perception and awareness from 657 confidential self-reports. BMC Womens Health. 2001;1(1):3. 
16. Arentz S, et al. A survey of the use of complementary medicine by a selfselected community group of Australian women with polycystic ovary syndrome. BMC Complement Altern Med. 2014;14(1):472.

17. Raja-Khan NES-V, Wu XK, Legro RS. The physiological basis of complementary and alternative medicines for polycystic ovary syndrome. Am J Physiol Endocrinol Metab. 2011;301(1):E1-E10.

18. Amini L, Tehranian N, Movahedin M, Tehrani FR, Ziaee S. Antioxidants and management of polycystic ovary syndrome in Iran: a systematic review of clinical trials. Iran J Reprod Med. 2015;13(1):1.

19. Arentz S, Smith CA, Abbott JA, Bensoussan A, et al. Herbal medicine for the management of polycystic ovary syndrome (PCOS) and associated oligo/ amenorrhoea and hyperandrogenism; a review of the laboratory evidence for effects with corroborative clinical findings. BMC Complement Altern Med. 2014;14(1):511

20. Zhang J, Li T, Zhou L, Tang L, Xu L, Wu T, Lin D. Chinese herbal medicine for subfertile women with polycystic ovarian syndrome. Cochrane Database Syst Rev. 2010;9:CD007535.

21. Murri M, Luque-Ramírez M, Insenser M, Ojeda-Ojeda M, Escobar-Morreale H, Hector F. Circulating markers of oxidative stress and polycystic ovary syndrome (PCOS): a systematic review and meta-analysis. Hum Reprod Update. 2013; 10.1093/humupd/dms059.

22. Ji Y, Tan S, Xu Y, Chandra A, Shi C, Song B, Qin J, Gao Y. Vitamin B supplementation, homocysteine levels, and the risk of cerebrovascular disease a meta-analysis. Neurology. 2013;81(15):1298-307.

23. Lai L, Li X, Flower A, Moore M, Liu J, Lewith G. Chinese herbal medicine for oligomenorrhoea and amenorrhoea in polycystic ovary syndrome: A systematic review and meta-analysis. J Alternat Complement Med. 2014; 20(5):A129-A129.

24. Lai L, Flower A, Prescott P, Moore M, Lewith $G$. Chinese herbal medicine for oligomenorrhoea and amenorrhoea in polycystic ovary syndrome: a randomised feasibility study in the United Kingdom. In: European journal of integrative medicine; 2015. p. 681-2.

25. Bazarganipour F, Taghavi SA, Montazeri A, Ahmadi F, Chaman R, Khosravi A. The impact of polycystic ovary syndrome on the healthrelated quality of life: a systematic review and meta-analysis. Iran J Reprod Med. 2015;13(2):61.

26. ESHRE. Revised 2003 consensus on diagnostic criteria and long-term health risks associated with polycystic ovary syndrome. Fertil Steril. 2004;81(1):19-25.

27. Azziz R, Carmina E, Dewailly D, Diamanti-Kandarakis E, Escobar-Morreale HF, Futterweit W, Janssen OE, Legro RS, Norman RJ, Taylor AE. The androgen excess and PCOS society criteria for the polycystic ovary syndrome: the complete task force report. Fertil Steril. 2009;91(2):456-88.

28. Wieland LS, Manheimer E, Berman BM. Development and classification of an operational definition of complementary and alternative medicine for the Cochrane collaboration. Altern Ther Health Med. 2011;17(2):50.

29. Higgins, J., Cochrane handbook for systematic reviews of interventions version 5.1. 0 (Updated on 2011). Cochrane Collab. www. cochrane-handbook.org. Accessed Jan, 2015: p. 164-166.

30. Cochrane. Review manager (RevMan). Copenhagen: The Cochrane Collaboration; 2012.

31. Artini $P G$, et al. Endocrine and clinical effects of myo-inositol administration in polycystic ovary syndrome. A randomized study. Gynecol Endocrinol. 2013;29(4):375-9.

32. Ardabili HR, Gargari BP, Farzadi L. Vitamin D supplementation has no effect on insulin resistance assessment in women with polycystic ovary syndrome and vitamin D deficiency. Nutr Res. 2012;32(3):195-201.

33. Chan CC, Koo MW, Ng EH, Tang OS, Yeung WS, Ho PC. Effects of Chinese green tea on weight, and hormonal and biochemical profiles in obese patients with polycystic ovary syndrome-a randomized placebo-controlled trial. J Soc Gynecol Investig. 2006;13(1):63-8

34. Costantino D, Minozzi G, Minozzi E, Guaraldi C. Metabolic and hormonal effects of myo-inositol in women with polycystic ovary syndrome: a doubleblind trial. Eur Rev Med Pharmacol Sci. 2009;13(2):105-10.

35. Cussons AJ, Watts GF, Mori TA, Stuckey BG. Omega-3 fatty acid supplementation decreases liver fat content in polycystic ovary syndrome: a randomized controlled trial employing proton magnetic resonance spectroscopy. J Clin Endocrinol Metab. 2009;94(10):3842-8.

36. Dona G, Sabbadin C, Fiore C, Bragadin M, Giorgino FL, Ragazzi E, et al. Inositol administration reduces oxidative stress in erythrocytes of patients with polycystic ovary syndrome. Eur J Endocrinol. 2012;166(4):703-10.
37. Gerli S, Mignosa M, Di Renzo GC. Effects of inositol on ovarian function and metabolic factors in women with PCOS: a randomized double blind placebo-controlled trial. Eur Rev Med Pharmacol Sci. 2003;7(6):151-9.

38. Gerli SEP, Ferrari A, Di Renzo AGC. Randomized, double blind placebocontrolled trial: effects of myo-inositol on ovarian function and metabolic factors in women with PCOS. Eur Rev Med Pharmacol Sci. 2007;11(5):347-54

39. Grant P. Spearmint herbal tea has significant anti-androgen effects in polycystic ovarian syndrome. A randomized controlled trial. Phytother Res. 2010;24(2):186-8.

40. Hosseinzadeh F, Hosseinzadeh-Attar MJ, Yekaninejad MS, Rashidi B. Effects of selenium supplementation on glucose homeostasis and free androgen index in women with polycystic ovary syndrome: a randomized, double blinded, placebo controlled clinical trial. J Trace Elem Med Biol. 2016;34:56-61.

41. Iuorno MJ, Jakubowicz DJ, Baillargeon J-P, Dillon P, Gunn RD, Allan G, Nestler JE. Effects of d-chiro-inositol in lean women with the polycystic ovary syndrome. Endocr Pract. 2002;8(6):417-23.

42. Kort DH, Lobo RA. Preliminary evidence that cinnamon improves menstrual cyclicity in women with polycystic ovary syndrome: a randomized controlled trial. Am J Obstet Gynecol. 2014:211(5):487.e1-6.

43. Lucidi RS, Thyer AC, Easton CA, Holden AE, Schenken RS, Brzyski RG. Effect of chromium supplementation on insulin resistance and ovarian and menstrual cyclicity in women with polycystic ovary syndrome. Fertil Steril. 2005;84(6):1755-7.

44. Mohammadi E, Rafraf M, Farzadi L, Asghari-Jafarabadi M, Sabour S. Effects of omega-3 fatty acids supplementation on serum adiponectin levels and some metabolic risk factors in women with polycystic ovary syndrome. Asia Pac J Clin Nutr. 2012;21(4):511-8.

45. Nestler JE, Jakubowicz DJ, Reamer P, Gunn RD, Allan G. Ovulatory and metabolic effects of D-chiro-inositol in the polycystic ovary syndrome. N Engl J Med. 1999;340(17):1314-20.

46. Raja-Khan N, Shah J, Stetter CM, Lott MEJ, Kunselman AR, Dodson WC, Legro RS. High-dose vitamin D supplementation and measures of insulin sensitivity in polycystic ovary syndrome: a randomized, controlled pilot trial. Fertil Steril. 2014;101(6):1740-6.

47. Rashidi B, Haghollahi F, Shariat FM, Zayerii F. The effects of calcium-vitamin $D$ and metformin on polycystic ovary syndrome: a pilot study. Taiwan J Obstet Gynecol. 2009;48(2):142-7.

48. Razavi M, Jamilian M, Kashan ZF, Heidar Z, Mohseni M, Ghandi Y, Bagherian $T$, Asemi Z. Selenium supplementation and the effects on reproductive outcomes, biomarkers of inflammation, and oxidative stress in women with polycystic ovary syndrome. Horm Metab Res. 2015;47:1-7.

49. Shahin AY, Mohammed SA. Adding the phytoestrogen Cimicifugae Racemosae to clomiphene induction cycles with timed intercourse in polycystic ovary syndrome improves cycle outcomes and pregnancy rates-a randomized trial. Gynecol Endocrinol. 2014;30(0):1-6.

50. Tehrani HG, Mostajeran F, Shahsavari S. The effect of calcium and vitamin D supplementation on menstrual cycle, body mass index and hyperandrogenism state of women with poly cystic ovarian syndrome. J Res Med Sci. 2014;19:875-80.

51. Vargas ML, Almario RU, Buchan W, Kim K, Karakas SE. Metabolic and endocrine effects of long-chain versus essential omega-3 polyunsaturated fatty acids in polycystic ovary syndrome. Metabolism. 2011;60(12):1711-8.

52. Wang JG, Anderson RA, Graham GM, Chu MC, Sauer MV, Guarnaccia MM, Lobo RA. The effect of cinnamon extract on insulin resistance parameters in polycystic ovary syndrome: a pilot study. Fertil Steril. 2007;88(1):240-3.

53. Kilicdag EB, Bagis T, Tarim TE, Aslan E, Erkanli S, Simsek E, Haydardedeoglu B, Kuscu E. Administration of B-group vitamins reduces circulating homocysteine in polycystic ovarian syndrome patients treated with metformin: a randomized trial. Hum Reprod. 2005;20(6):1521-8.

54. Jamilian $\mathrm{M}$, et al. Comparison of myo-inositol and metformin on clinical, metabolic and genetic parameters in polycystic ovary syndrome: a randomized controlled clinical trial. Clin Endocrinol. 2017;87(2):194-200.

55. Mehta G, Sen S. Fine tuning the hydrophilic-hydrophobic balance in inositols through annulation: an analysis of the hydrogen-bonded architectures of'annulated inositols'. CrystEngComm. 2005;2005(7):656-63.

56. Dunaif A, Book CB. Insulin resistance in the polycystic ovary syndrome. Clin Res Diabetes Obes. 1997;2:249.

57. Larner J. D-chiro-inositol-its functional role in insulin action and its deficit in insulin resistance. J Diabetes Res. 2002;3(1):47-60. 
58. Nestler JE, Jakubowicz DJ, Falcon A, de Vargas AF, Brik C, Quintero N, Medina F. Insulin stimulates testosterone biosynthesis by human Thecal cells from women with polycystic ovary syndrome by activating its own receptor and using Inositolglycan mediators as the signal transduction system 1. J Clin Endocrinol Metab. 1998;83(6):2001-5.

59. Arendrup K, Gregersen G, Hawley J, Hawthorne JN. High-dose dietary myo-inositol supplementation does not alter the ischaemia phenomenon in human diabetics. Acta Neurol Scand. 1989;80(2):99-102.

60. Bishop FL, Yardley L, Lewith GT. A systematic review of beliefs involved in the use of complementary and alternative medicine. J Health Psychol. 2007; 12(6):851-67.

Submit your next manuscript to BioMed Central and we will help you at every step:

- We accept pre-submission inquiries

- Our selector tool helps you to find the most relevant journal

- We provide round the clock customer support

- Convenient online submission

- Thorough peer review

- Inclusion in PubMed and all major indexing services

- Maximum visibility for your research

Submit your manuscript at www.biomedcentral.com/submit 